PAPER • OPEN ACCESS

Impact of new operational dosimetric quantities on individual monitoring services

To cite this article: M Caresana et al 2021 J. Radiol. Prot. 411110

View the article online for updates and enhancements.

Fast and reliable detection of any increase in dose rate in the workplace 


\title{
Impact of new operational dosimetric quantities on individual monitoring services
}

\author{
M Caresana ${ }^{1, *}(\mathbb{D})$, L Garlati ${ }^{1}$, G Zorloni $^{1}$, R Behrens ${ }^{2}$ (D), \\ T Otto $^{3}{ }^{(D}$, G Minchillo $^{4}$ and F Rossi ${ }^{5}$ \\ ${ }^{1}$ Politecnico di Milano-Department of Energy, Via Lambruschini 4, 20156 Milano, \\ Italy \\ 2 Physikalisch-Technische Bundesanstalt (PTB), Bundesallee 100, D-38116 \\ Braunschweig, Germany \\ ${ }^{3}$ CERN, CH-1211 Geneve 23, Switzerland \\ ${ }^{4}$ European Commission-Joint Research Centre (JRC), Via E. Fermi 2749, 21027 \\ Ispra (VA), Italy \\ 5 Fisica Sanitaria AOU Careggi, Largo G.A. Brambilla 3, 50134 Firenze, Italy \\ E-mail: marco.caresana@polimi.it
}

Received 22 March 2021; revised 8 June 2021

Accepted for publication 22 June 2021

Published 15 November 2021

\section{Abstract}

The new operational dosimetric quantities framework, proposed in the ICRU95 report, has stimulated the scientific community to start investigations that aim to assess its impact on radiation protection practices. As part of this effort, the present study describes an inter-comparison exercise among individual monitoring services (IMSs) on passive whole-body dosimetry. The inter-comparison is performed in terms of both the existing operational dose quantity $H_{\mathrm{p}}(10)$ and its proposed replacement $H_{\mathrm{p}}$, to allow an evaluation of the actions that may be necessary to adapt dosimetry systems to the proposed quantity. For two of the tested IMSs, simple modifications to the detector response function, or the dose calculation algorithm, were sufficient to obtain results within acceptable limits. However, these approaches are not sufficient to give a level of

\footnotetext{
* Author to whom any correspondence should be addressed.
}

Original Content from this work may be used under the terms of the Creative Commons Attribution 4.0 licence. Any further distribution of this work must maintain attribution to the author(s) and the title of the work, journal citation and DOI. 
performance comparable to that achieved in terms of $H_{\mathrm{p}}(10)$. This may require a modification to dosemeter design.

Keywords: operational quantities, personal dosimetry, dosimetry service.

(Some figures may appear in colour only in the online journal)

\section{Introduction}

In 2017 the International Commission on Radiation Units and Measurements (ICRU) and the International Commission on Radiological Protection (ICRP) issued a draft for consultation containing a novel approach to external radiation exposure based on the introduction of new operational dosimetric quantities. These quantities include ambient dose $H^{*}$ and personal dose $H_{\mathrm{p}}$ for environmental and whole-body personal dosimetry, respectively. There are also new quantities for skin and eye lens dosimetry that are expressed in terms of absorbed dose instead of equivalent dose. The report based on the 2017 draft was published in late 2020 as ICRU Report 95 [1]. The main motivation was to tackle issues with the existing operational dosimetric quantities. In some irradiation situations, for instance in high-energy radiation fields, the operational quantities are not conservative with respect to the limit quantities such as effective dose. In other situations, such as low-energy photons in the energy range of tens of $\mathrm{keV}$, the operational quantities are overly conservative. A detailed description of the proposed new framework has been provided by Otto et al [2]. Unlike the current operational quantity for whole-body personal dosimetry $H_{\mathrm{p}}(d)$, the newly proposed quantity $H_{\mathrm{p}}$, does not relate to a specific depth in tissue. It is instead based on effective dose and is calculated across the whole energy spectrum using an anthropomorphic computational phantom [3]. For calibration of dosemeters, the same conversion coefficients can be used on surrogates of the computational phantoms, such as the ISO water slab phantoms or the ISO PMMA (polymethyl methacrylate) rod phantom [4] [5], without important variations [1]. However, the proposed change to $H_{\mathrm{p}}$ implies a variation in a dosemeter's response because of the change in conversion coefficients. References [6-8] report theoretical studies of how a change to $H_{\mathrm{p}}$ would affect the responses of existing photon dosemeters. As expected, these studies show that the responses of the dosemeters are not suitable for the new quantity below an energy of $50 \mathrm{keV}$.

The new operational quantities will have a significant impact in terms of new procedures, education of personnel, adaption of calibration facilities, re-characterisation of dosimetry systems and modification of existing dosemeters. In this view, EURADOS [9] set up a task group that aimed to prepare a report on the consequence of the introduction of the new quantities on radiation protection practices. Within the framework of this effort, this paper focuses on an experimental comparison between $H_{\mathrm{p}}(10)$ and $H_{\mathrm{p}}$, describing an inter-comparison exercise among individual monitoring services (IMSs) in terms of both quantities. In particular, two participants in the standard (i.e. referring to the existing quantities) inter-comparison exercise, agreed to characterise their dose evaluation algorithm in terms of $H_{\mathrm{p}}$, based on the conversion coefficients reported in [8]. The results in terms of existing operational quantities and in terms of $H_{\mathrm{p}}$ have been processed according to the standard ISO 14146 [10].

\section{Inter-comparison organisation}

The whole-body dosimetry inter-comparison has been organised by the laboratory of Metrology of Ionizing Radiation (LaMIR) at the Department of Energy of the Politecnico di Milano. 
Table 1. Radiation qualities used for the inter-comparison exercise.

\begin{tabular}{lll}
\hline Radiation quality & Irradiation angle & Dose range $(\mathrm{mSv})$ \\
\hline $\mathrm{W}-60$ & $0^{\circ}$ & $1.6-2.4$ \\
$\mathrm{~W}-80$ & $0^{\circ}$ & $0.8-1.2$ \\
$\mathrm{~W}-110$ & $0^{\circ}$ & $1.6-2.4$ \\
$\mathrm{~W}-110$ & $45^{\circ}$ & $5.5-7.0$ \\
$\mathrm{~W}-300$ & $0^{\circ}$ & $1.6-2.4$ \\
S-Cs & $0^{\circ}$ & $0.4-0.6$ \\
S-Cs & $0^{\circ}$ & $1.2-1.8$ \\
S-Cs & $0^{\circ}$ & $4.0-6.0$ \\
S-Cs & $0^{\circ}$ & $40.0-60.0$ \\
S-Cs+W-60 & $0^{\circ}$ & $0.8-1.2$ \\
\hline
\end{tabular}

a The dose in the mixed field irradiation is equally distributed between the two irradiation qualities.

It has been carried out as part of the process leading to the accreditation of the LaMIR as the proficiency test provider. Seven IMSs participated in the inter-comparison exercise, with a total of nine dosemeter sets (an IMS could participate with more than one dosemeter type) and the quantities used were $H_{\mathrm{p}}(10)$ and $H_{\mathrm{p}}(0.07)$, both on the ISO water slab phantom. The participants used two different dosemeter types:

- Two dosimetry systems based on a film-badge;

- Seven dosimetry systems based on TL (thermoluminescence) detectors;

Each dosimetry system is identified by a letter from A to L (leaving out I, J and K).

The irradiation scheme is reported in table 1 . The dose ranges indicated in table 1 were given to the irradiation laboratory, but were unknown to the participants. The participants expected the irradiation in a dose range $0.4-60 \mathrm{mSv}$, but had no knowledge of the association between energy and dose range.

A graphical representation of the IMSs performance in terms of $H_{\mathrm{p}}(10)$ is shown in figure 1 . Each tested dosimetry system is indicated by a letter. The comparison is presented as response $R$, i.e. the dose measured by the IMS divided by the reference dose certified by the irradiation laboratory. The continuous lines indicate the acceptance limits as proposed in the standard ISO 14146 [10]. Only two dosimetry systems, namely E and F, present underestimations lower than the minimum acceptance limit, for a number of experimental results greater than $10 \%$ of the total (the maximum allowed by ISO 14 146). Figure 2 reports the results in terms of $H_{\mathrm{p}}(0.07)$ and confirms the underestimation of the above indicated dosimetry systems. The graph shown in figure 3 highlights the systematic deviation by specific radiation quality. In this case the almost flat response in terms of $H_{\mathrm{p}}(10)$ indicates that, on average, the response of the dosimetry systems is energy independent.

\section{Data analysis in terms of $H_{\mathrm{p}}$}

When moving from the existing operational dosimetric quantities to $H_{\mathrm{p}}$, the impact on the dosimetry systems can be identified in one of the following scenarios, sorted by increasing severity: 


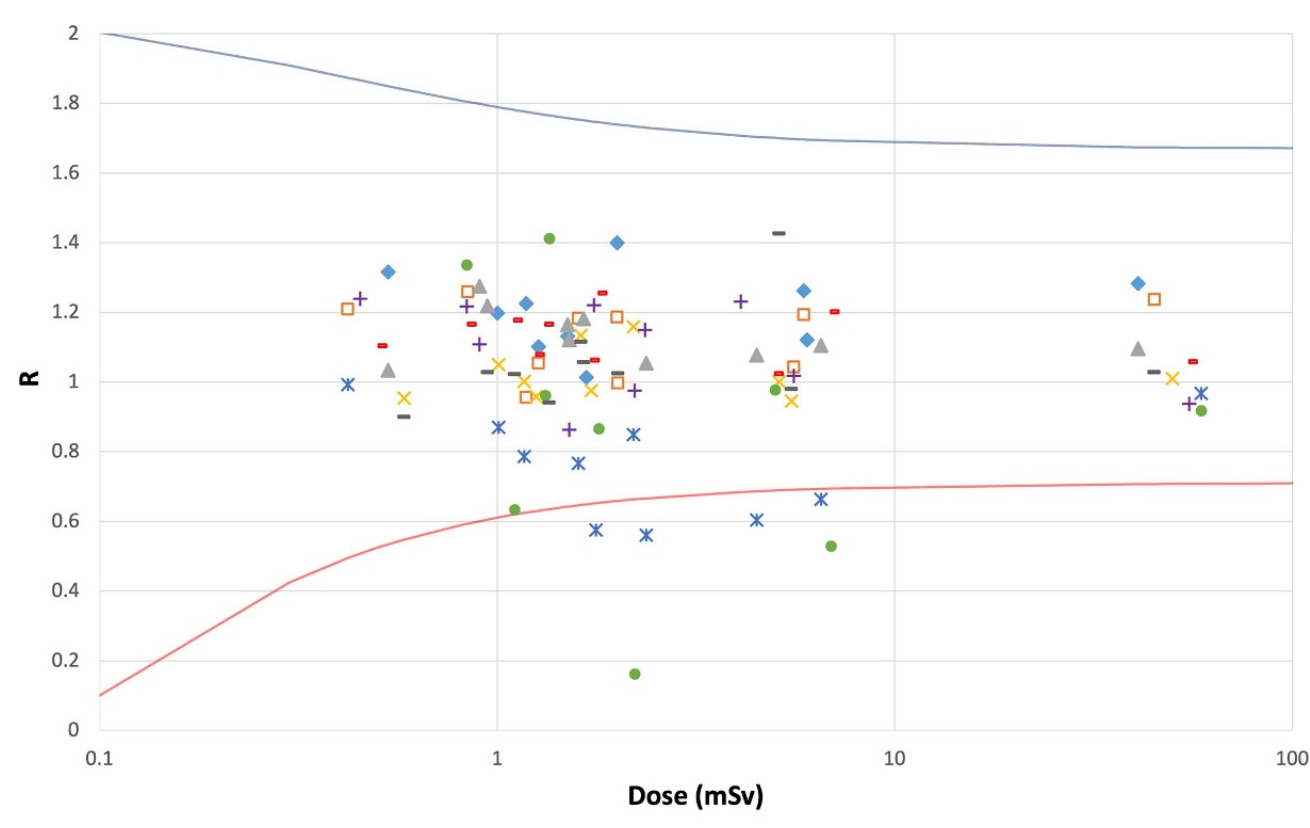

$\rightarrow$ A $\square \triangle \mathbf{B} \times \mathbf{D} * \mathbf{E} \bullet \mathbf{F}+\mathbf{G}=\mathbf{H}-\mathbf{L}-\mathbf{M i n}-\mathbf{M a x}$

Figure 1. Results of the inter-comparison in terms of $H_{\mathrm{p}}(10)$. The ordinate shows the ratio $R$ of the dose measured by the IMS, normalised to the reference dose of the irradiation.

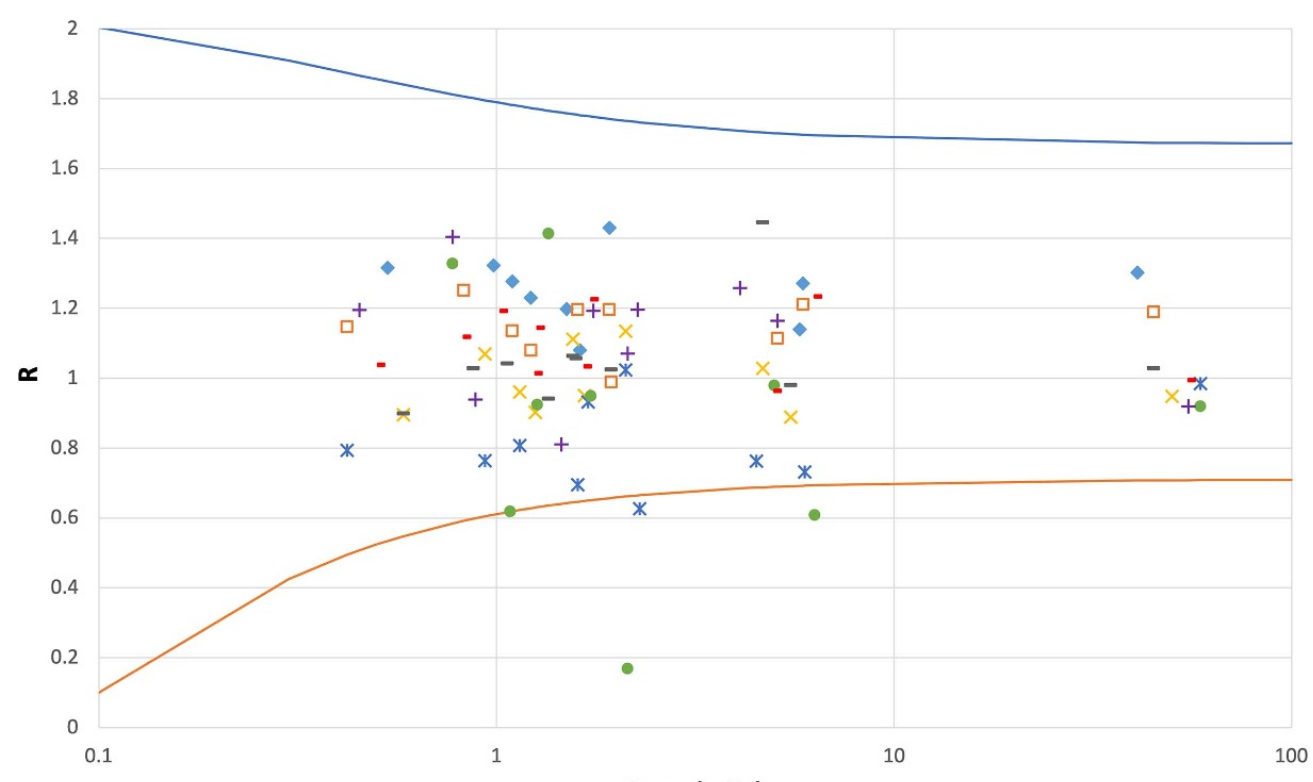

Dose (mSv)

- A $\square$ B C $\times$ D $*$ E $\bullet F+G-H-L-M i n-M a x$

Figure 2. Results of the inter-comparison in terms of $H_{\mathrm{p}}(0.07)$. The ordinate shows the ratio $R$ of the dose measured by the IMS, normalised to the reference dose of the irradiation. 


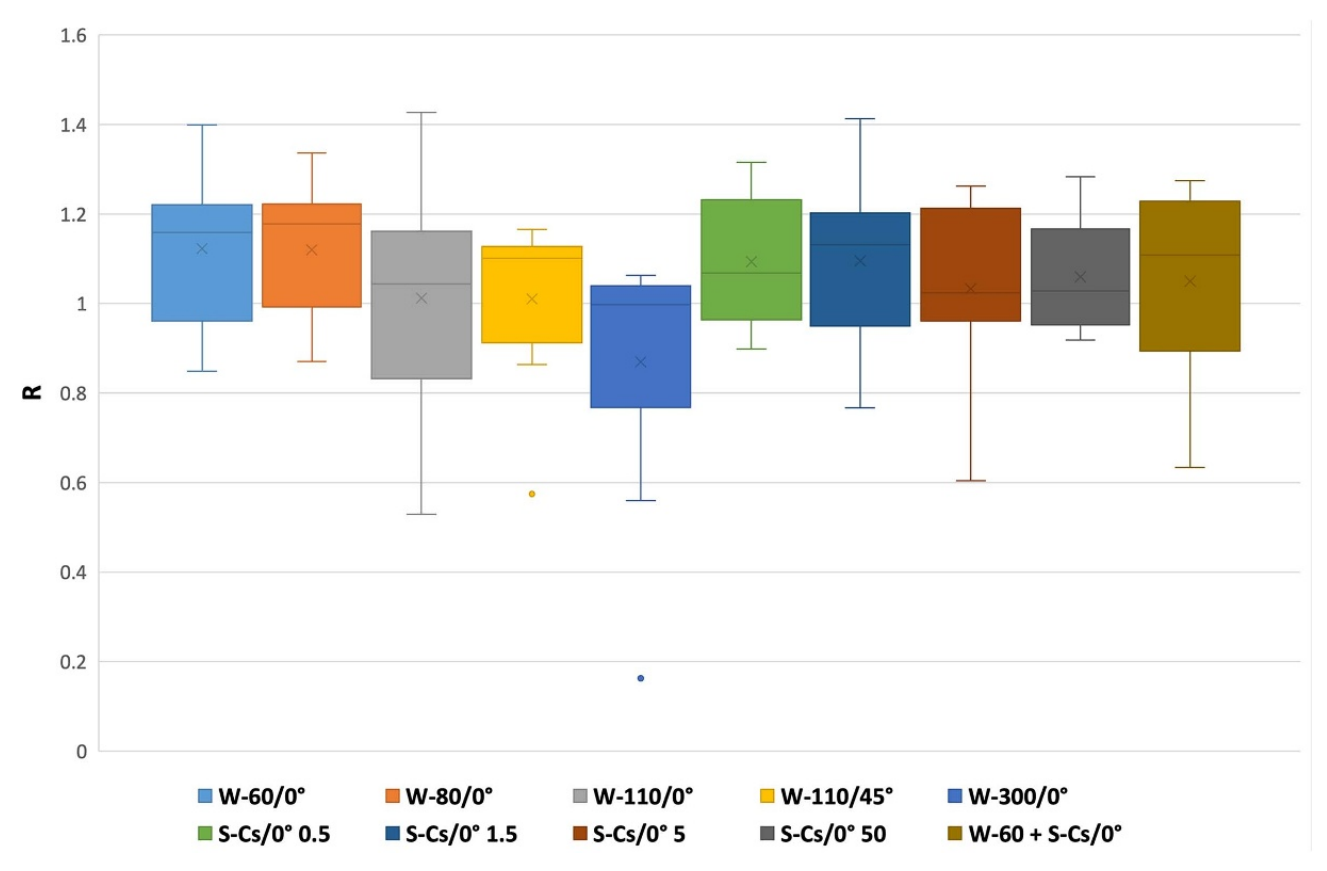

Figure 3. A box plot representation of the results of the inter-comparison in terms of $H_{\mathrm{p}}(10)$. The ratio $\mathrm{R}$ is grouped per radiation quality, assembling the results of all the IMSs. The line inside boxes is the median, the $\mathrm{x}$ is the mean, the box is the interquartile range and the whiskers represent the 2 nd and the 98 th percentile. The points external to the whiskers indicate clearly wrong dosimetric results.

(a) The dosemeter responds well 'as it is', or by simply applying an energy-independent correction coefficient.

(b) The dosemeter needs a modification of the response function by changing the reference value of the radiation qualities, but the dose calculation algorithm also works well with the new response function.

(c) The algorithm of the dose calculation must be modified but the design of the badge also works well for $H_{\mathrm{p}}$.

(d) None of the above give a satisfactory response, in which case the specified energy range of the dosemeter must be restricted or a new dosemeter badge design is needed.

Scenario (i) is of course the desirable one, but very unlikely to occur. Scenarios (ii) and (iii) are manageable without a serious impact on the cost. At the other extreme, scenario (iv) will have a significant impact in terms of the time and cost involved in developing and commissioning a new dosemeter badge.

It is worth specifying that the analysis presented in this work is not exhaustive because the number of energies/irradiation and irradiation angles is limited and lower than the minimum needed for a complete type test. Also, the number of the tested dosemeter types is only a subset of the existing ones. Within this framework, the aim of our analysis is to identify which scenario is more likely to occur. Dosimetry systems E and F were excluded from this analysis because they did not provide results within the acceptance limits in the standard inter-comparison, i.e. in terms of $H_{\mathrm{p}}(10)$. 
Table 2. Conversion coefficients for total air kerma to personal dose $H_{\mathrm{p}}$, and for total air kerma to dose equivalent $H_{\mathrm{p}}(10)$ for radiation qualities of inter-comparison.

\begin{tabular}{lll}
\hline Radiation quality & $h_{\mathrm{p} K}(\alpha)$ & $h_{\mathrm{p} K}(10 ; \alpha)_{\text {slab }}$ \\
\hline $\mathrm{W}-60-0^{\circ}$ & $0.8906(0.8879)^{\mathrm{a}}$ & 1.55 \\
$\mathrm{~W}-80-0^{\circ}$ & 1.1772 & 1.77 \\
$\mathrm{~W}-110-0^{\circ}$ & 1.4021 & 1.87 \\
$\mathrm{~W}-110-45^{\circ}$ & 1.2042 & 1.72 \\
$\mathrm{~W}-300-0^{\circ}$ & 1.1545 & 1.47 \\
S-Cs- $0^{\circ}$ & 1.0158 & 1.21 \\
\hline
\end{tabular}

a The conversion coefficients are indicated for a distance of $2.5 \mathrm{~m}$ between the $\mathrm{x}$-ray tube and the point of irradiation; for a distance of $1.0 \mathrm{~m}$ we apply the values in brackets.

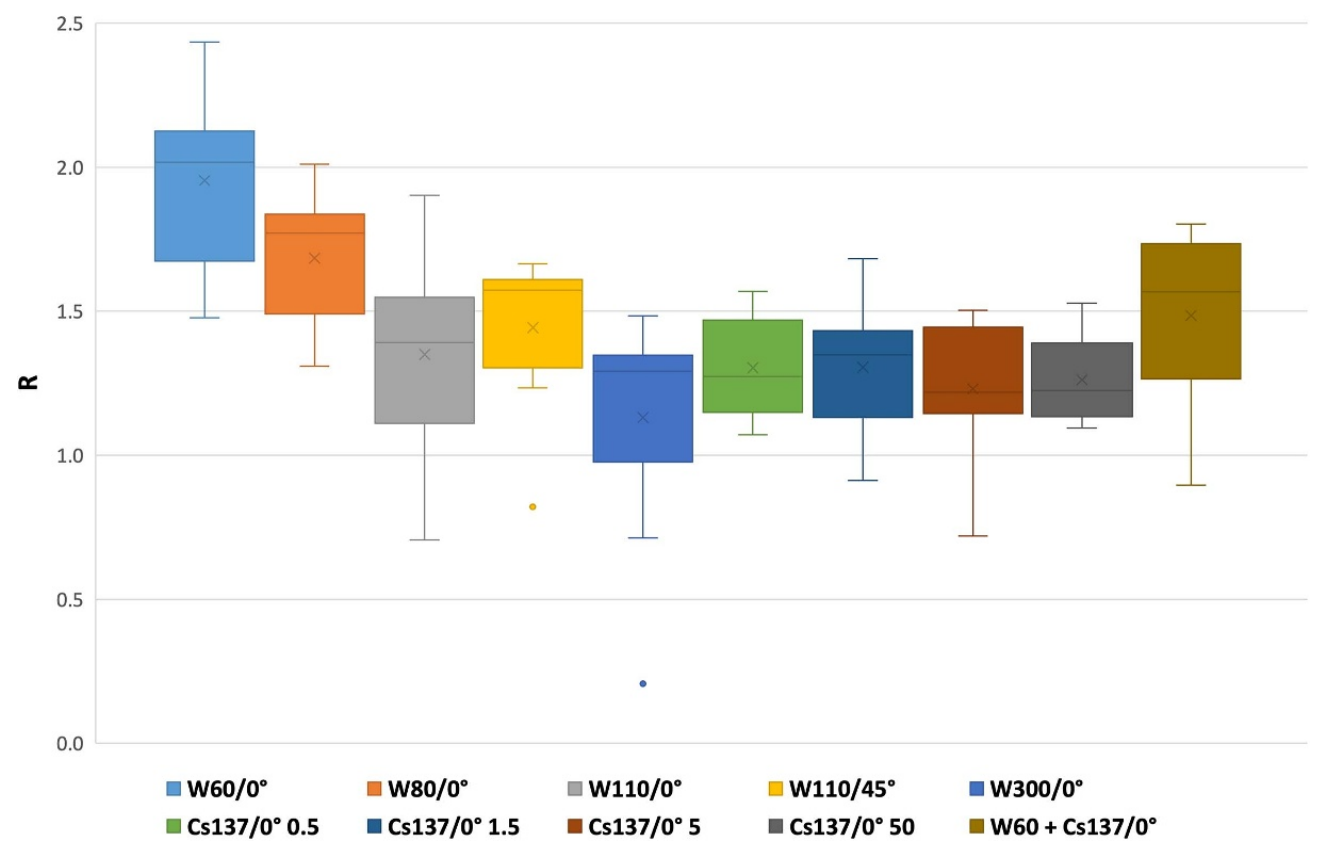

Figure 4. A box plot representation of the results of $H_{\mathrm{p}}$ without the normalisation coefficient. $\mathrm{R}$ is grouped per radiation quality, assembling the results of all the IMSs. The line inside boxes is the median, the $\mathrm{x}$ is the mean, the box is the interquartile range and the whiskers represent the $2 \mathrm{nd}$ and the 98 th percentile. The points external to the whiskers indicate clearly wrong dosimetric results. The overestimation is more pronounced at low energies.

To check scenario (i), the same representation has been chosen in the figures expressing the reference dose in terms of $H_{\mathrm{p}}$ and maintaining unchanged the results given by the IMS as $H_{\mathrm{p}}(10)$. The reference values for $H_{\mathrm{p}}$ were obtained by applying the conversion coefficients reported in table 2 and taken from [8]. As the new conversion coefficients $h_{\mathrm{p} K}(\alpha)$ are lower than the previous ones $h_{\mathrm{p} K}(10 ; \alpha)_{\mathrm{slab}}$, especially at low energies, the values are shifted to higher values of $R$, indicating an overestimation more pronounced at qualities W-60 and W-80 (figure 4). 


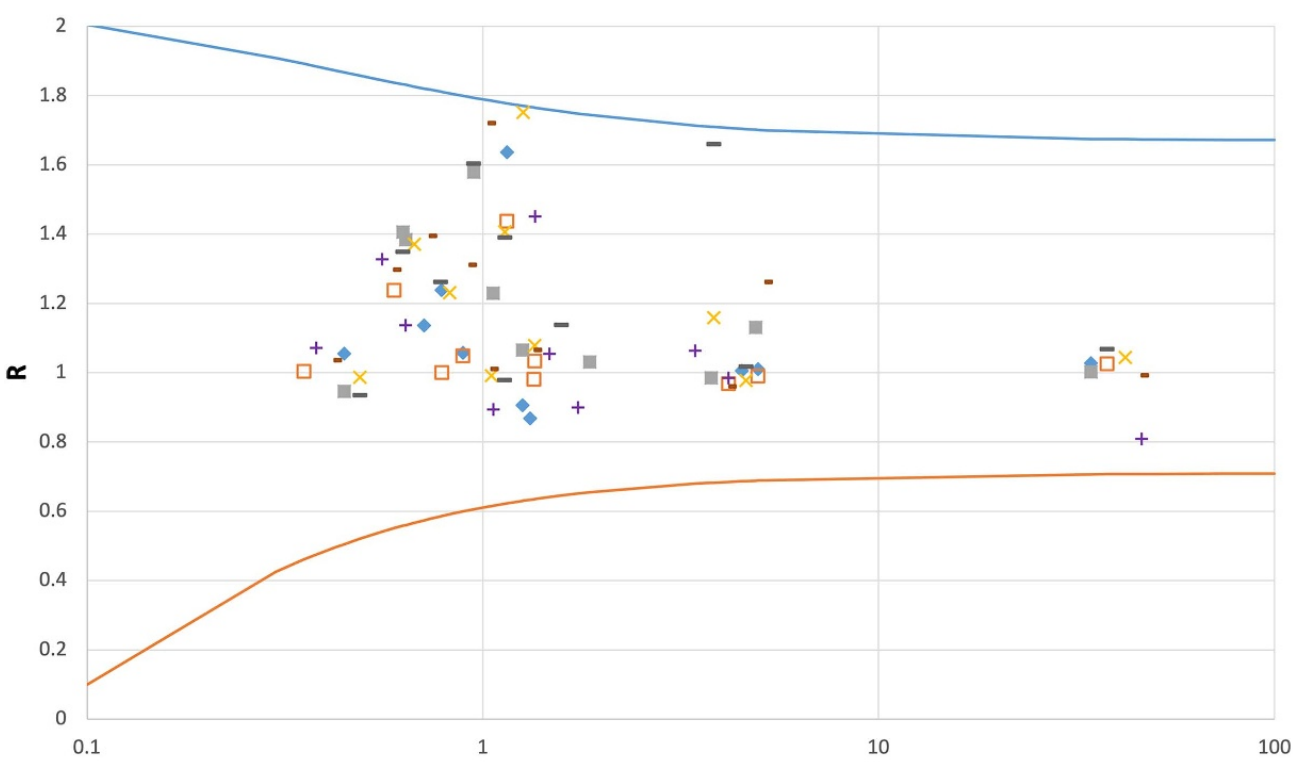

Dose (mSv)

- A $\square$ B $\square \mathbf{C} \times \mathbf{D}+\mathbf{G}-\mathbf{H}-\mathbf{L}-\mathrm{Min}-\mathrm{Max}$

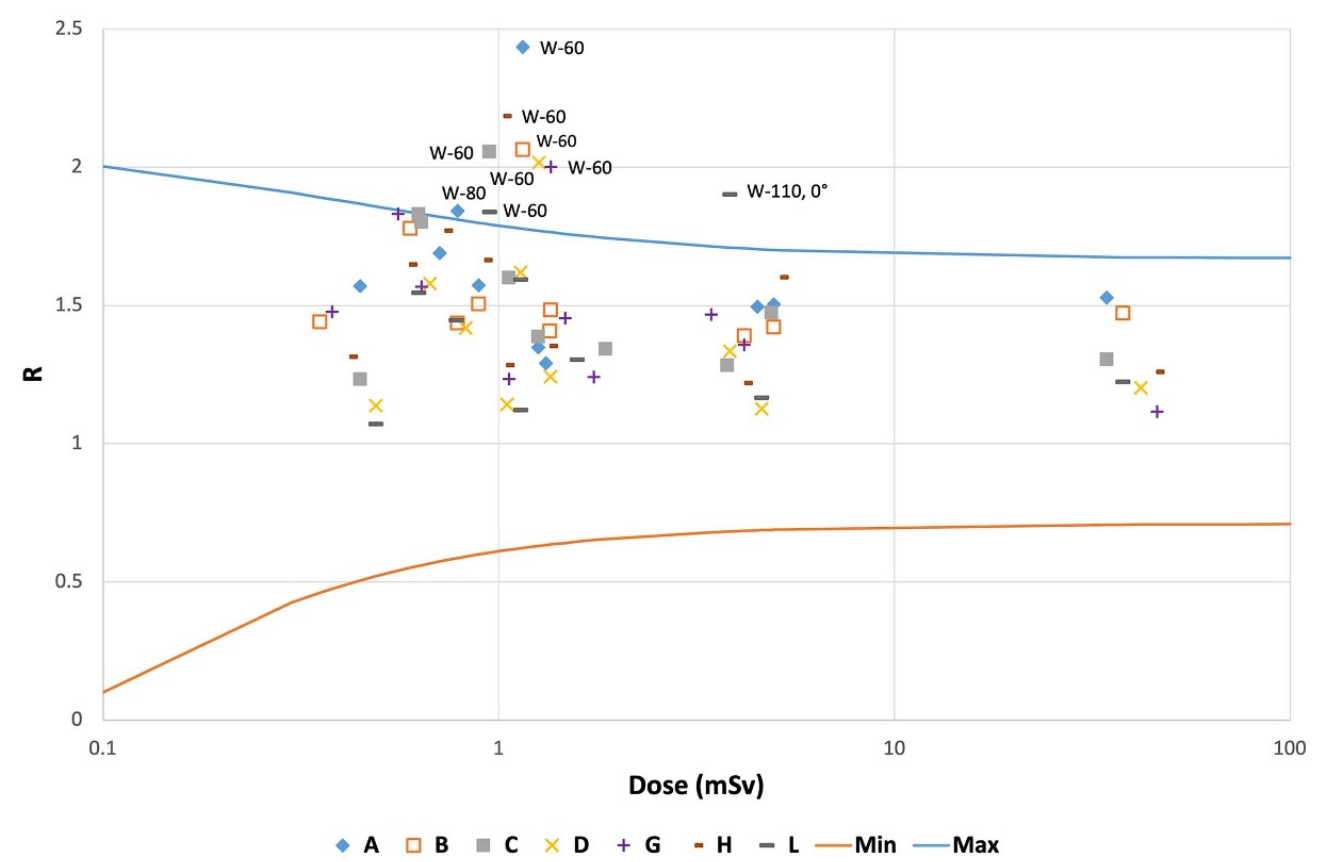

Figure 5. Results in terms of $H_{\mathrm{p}}$ with (top) and without (bottom) the energy-independent normalisation coefficient. 
Table 3. The Panasonic UD-802A badge: structure and composition.

\begin{tabular}{llll}
\hline Detector & Composition & $\begin{array}{l}\text { Overall front } \\
\text { filtration } \\
\left(\mathrm{mg} \mathrm{cm}^{-2}\right)\end{array}$ & $\begin{array}{l}\text { Overall rear } \\
\text { filtration } \\
\left(\mathrm{mg} \mathrm{cm}^{-2}\right)\end{array}$ \\
\hline 1 & ${ }^{n} \mathrm{Li}_{2} \mathrm{~B}_{4} \mathrm{O}_{7}: \mathrm{Cu}$ & plastic: 14 & plastic: 28 \\
2 & ${ }^{n} \mathrm{Li}_{2} \mathrm{~B}_{4} \mathrm{O}_{7}: \mathrm{Cu}$ & plastic: 303 & plastic: 303 \\
3 & $\mathrm{CaSO}_{4}: \mathrm{Tm}$ & plastic: 303 & plastic: 303 \\
4 & $\mathrm{CaSO}_{4}: \mathrm{Tm}$ & plastic: 143 —lead: 874 & plastic: 143 -lead: 874 \\
\hline
\end{tabular}

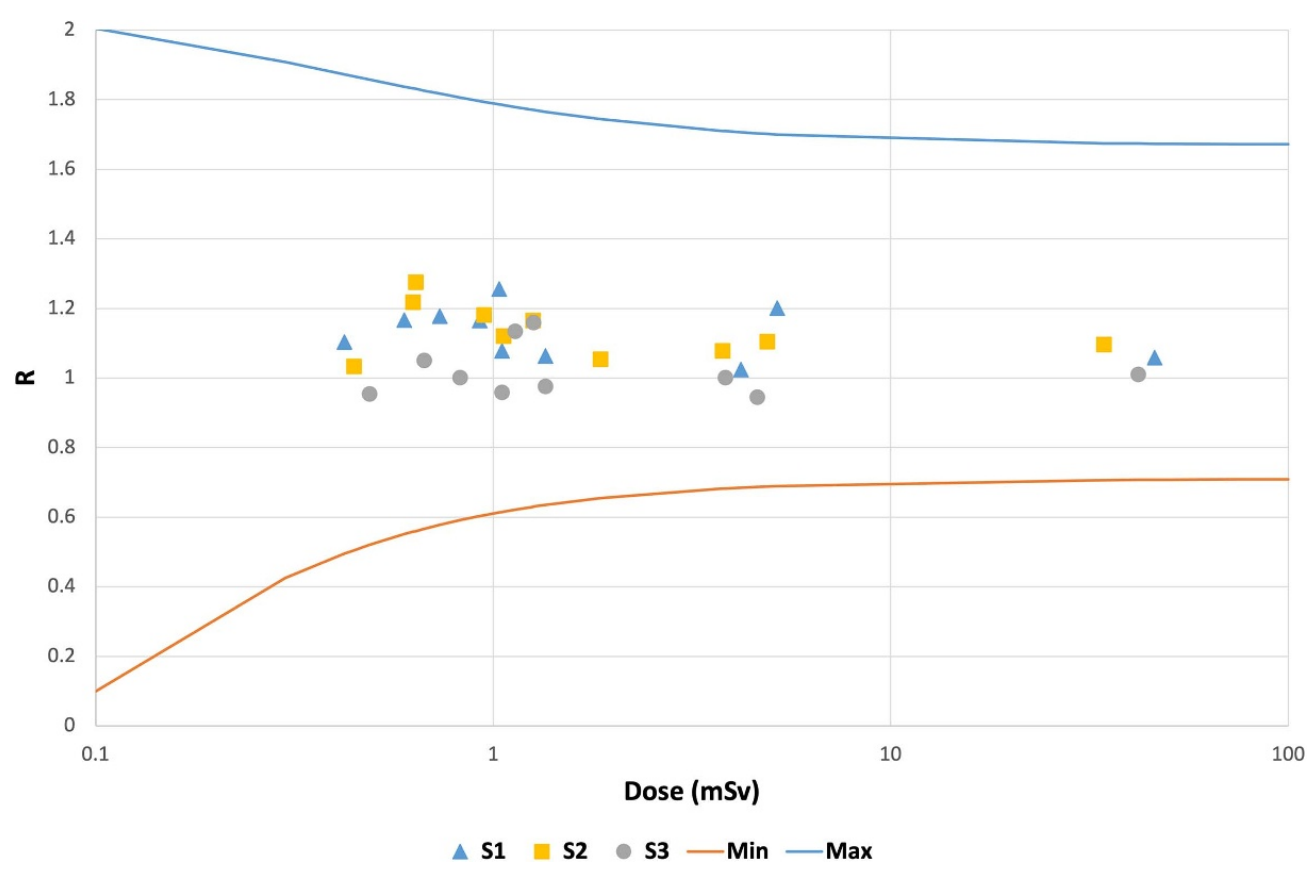

Figure 6. Inter-comparison results in terms of $H_{\mathrm{p}}(10)$ of the two selected IMSs.

The aim of a first attempt to double check the validity of scenario (i), without modification of either the dosemeter badge or its algorithm, was to identify a normalisation coefficient $C_{n}$, which was energy independent, to apply to the data referenced to $H_{\mathrm{p}}(10)$. If the normalisation coefficient exists it means that scenario (i) could be a valid option. $C_{n}$ was tuned in the range $1-1.5$, to get the mean value of $R$ referenced to $H_{\mathrm{p}}$ equal to one for $S-C s$ irradiation. In principle it is possible to also normalise to different energies, and probably get better results. However, in this paper the focus is more on the approach rather than fine tuning. Figure 5 compares the $R$ values with and without the normalisation coefficient. Even though the results are within the limit of the trumpet curves, the overestimation at low energies is still evident. Thus, scenario (i) can work for this specific inter-comparison exercise but, as several points are rather near the upper limit, it does not seem a reliable approach.

A second more detailed analysis directly involved the two IMSs that recalculated the response function of their dosimetry systems based on the coefficients reported in [8] and consequently optimised the algorithm, as discussed below. This kind of analysis allows one 


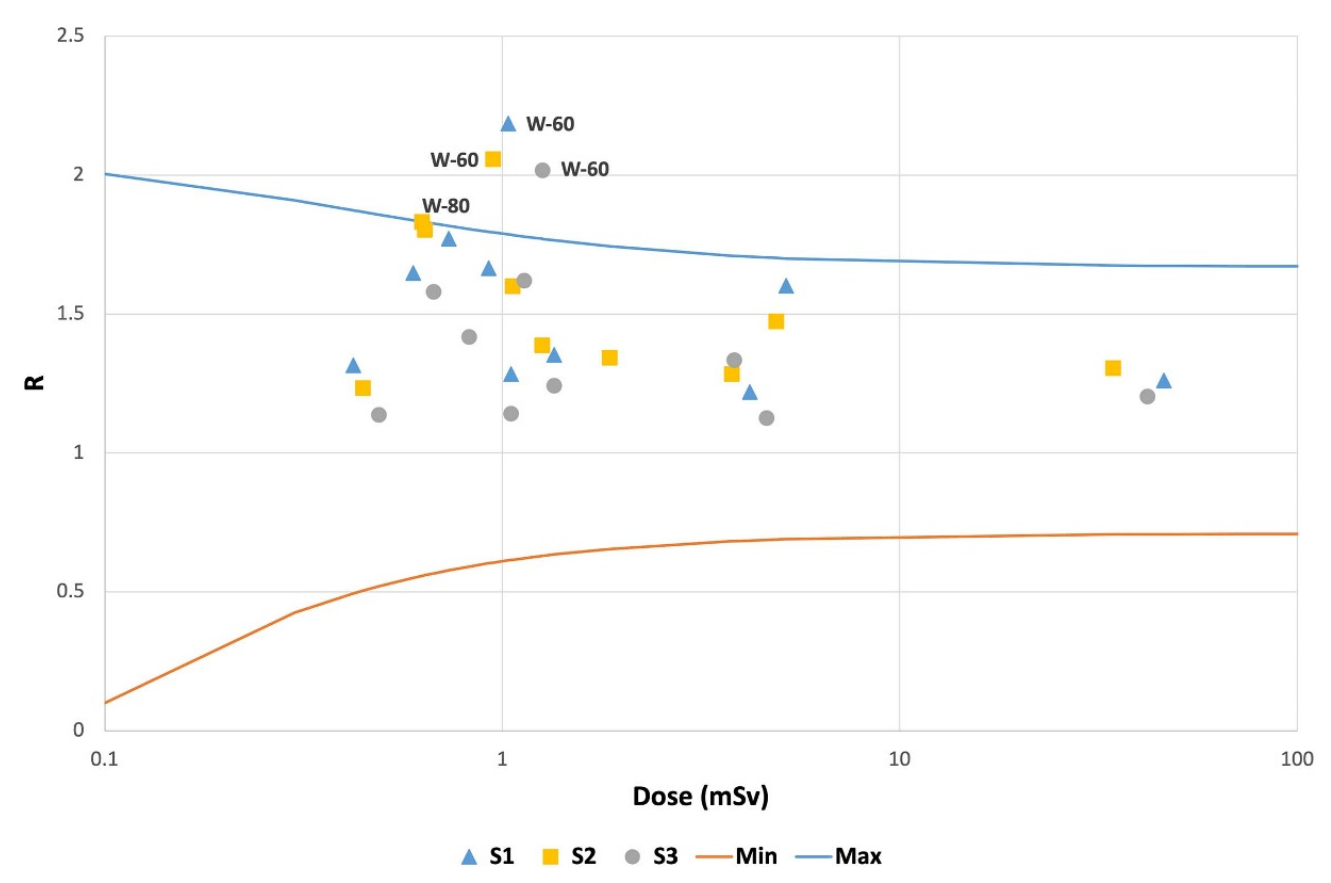

Figure 7. Inter-comparison results in terms of $H_{\mathrm{p}}(10)$, referenced to $H_{\mathrm{p}}$, of the two selected IMSs.

to check if scenario (ii) and, partially, scenario (iii) are likely to occur. The first IMS participated in the inter-comparison with two identical sets of dosemeters (only different readers used). The dosemeter is composed of two detectors, both TLD-100 (Thermo Scientific Harshaw). One detector is covered by a thin $(0.2 \mathrm{~mm})$ plastic layer for protective purposes. The second detector is shielded by a $1.5 \mathrm{~mm}$ thick aluminium filter. With this configuration the ratio of the two readings is a monotonic function of photon energy. The dosemeter is characterised in terms of $H_{\mathrm{p}}(10)$ with reference irradiation at different beam qualities, typically $\mathrm{N}$ and $\mathrm{W}$ series and $\mathrm{S}-\mathrm{Cs}$, according to the ISO 4037. The response function is normalised to $\mathrm{S}-\mathrm{Cs}$. The new response function in terms of $H_{\mathrm{p}}$ has been obtained by re-scaling the reference irradiation with the new conversion coefficients. The personal dose is calculated by multiplying the response $\mathrm{D}$ of the filtered TL detector referred to as S-Cs by the response function in terms of $H_{\mathrm{p}}$.

The second IMS uses a whole-body dosemeter which is sensitive to beta, $X$ and $\gamma$ radiation. It consists of a transparent plastic outer casing (of dimensions $50 \times 50 \times 14 \mathrm{~mm}$ ) from the Panasonic TLD badges UD-802A series. The badge contains four thermoluminescent detectors covered with different protective materials and/or shielding. The choice of thickness and front and rear construction of the dosemeter serves the purpose of providing a more selective response to radiation of different nature and energy. Table 3 shows the composition of each thermoluminescent detector and the overall front and rear thickness of the different materials that shield each of them. The different elements and filtrations enable the discrimination of the radiation type through the ratio between the light counts of different elements. The personal dose equivalent is calculated using equation (1) that uses only signals from the 2nd and 3rd element. 


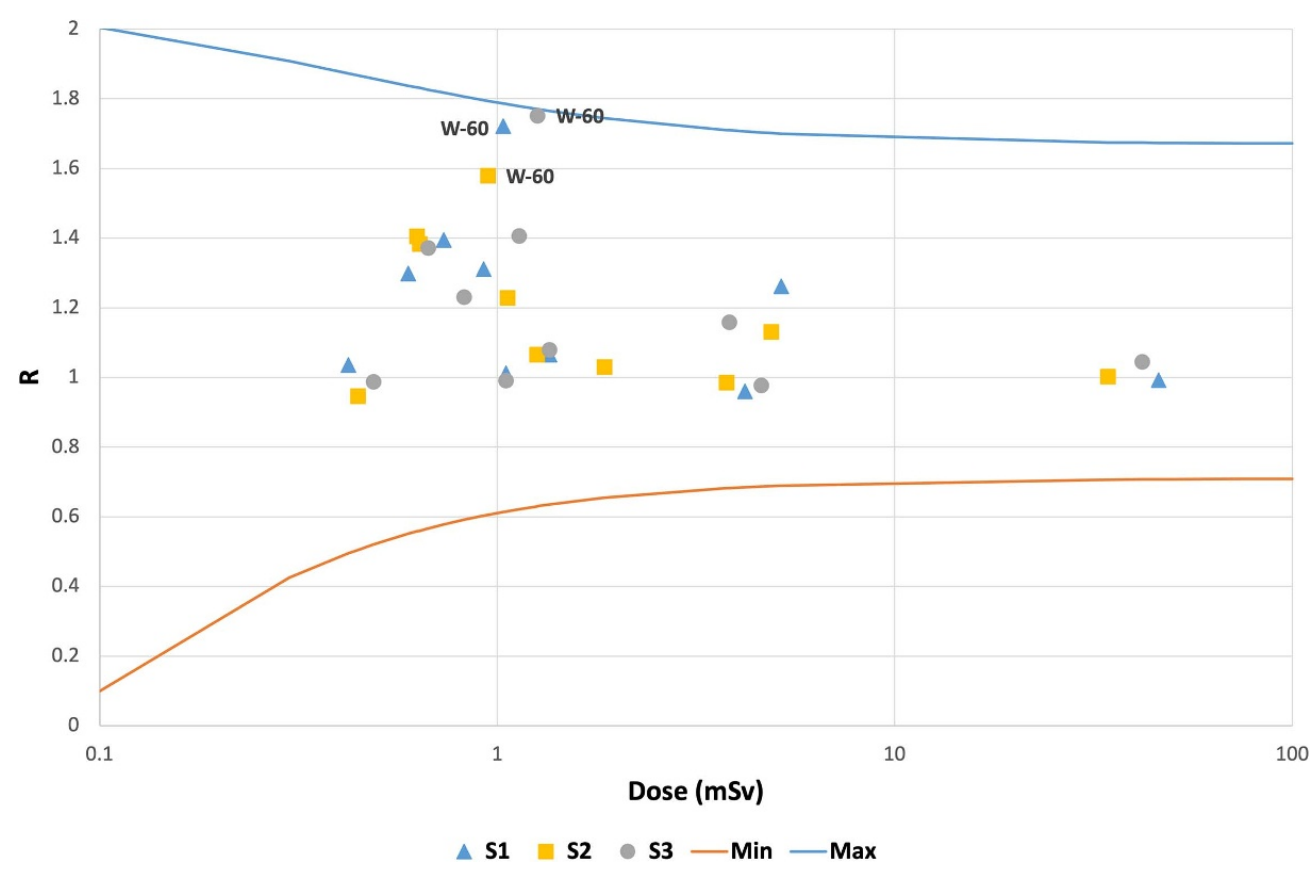

Figure 8. Inter-comparison results referenced to $H_{\mathrm{p}}$ and corrected with the energyindependent normalisation constant.

$$
D=\left(C_{2}-B_{2}\right) \cdot S_{\mathrm{Cs}-\mathrm{kerma}} \cdot F\left(\frac{C_{3}-B_{3}}{C_{2}-B_{2}}\right)
$$

where:

- $D$ is the personal dose equivalent $H_{\mathrm{p}}(10)$

- $C_{2}, C_{3}$ are the signals of the 2 nd and 3 rd element

- $B_{2}, B_{3}$ are the background signals of the 2 nd and 3 rd element

- $S_{\mathrm{Cs}-\text { kerma }}$ is the response function in terms of air kerma

- $\mathrm{F}\left(\frac{C_{3}-B_{3}}{C_{2}-B_{2}}\right)\left[\frac{S v}{G y}\right]$ is a fourth-order polynomial function correlating the air kerma to $H_{\mathrm{p}}(10)$

To move from $H_{\mathrm{p}}(10)$ to $H_{\mathrm{p}}$, the dosimetric algorithm keeps equation (1) unchanged but modifies the polynomial function. This approach can be regarded as a change in algorithm and is therefore connected to scenario (iii). According to the confidentiality rules of the intercomparison exercise, results of these three sets will be presented anonymously, using the codes $\mathrm{S} 1, \mathrm{~S} 2$ and $\mathrm{S} 3$.

To better understand the effect of the new dosimetric quantities, figure 6 shows the results of the regular inter-comparison, figure 7 shows the results in terms of $H_{\mathrm{p}}(10)$ compared with $H_{\mathrm{p}}$ as a reference, figure 8 shows the impact of the correction with the energy-independent normalisation constant, as described above, and figure 9 shows the results with the modified algorithm. 


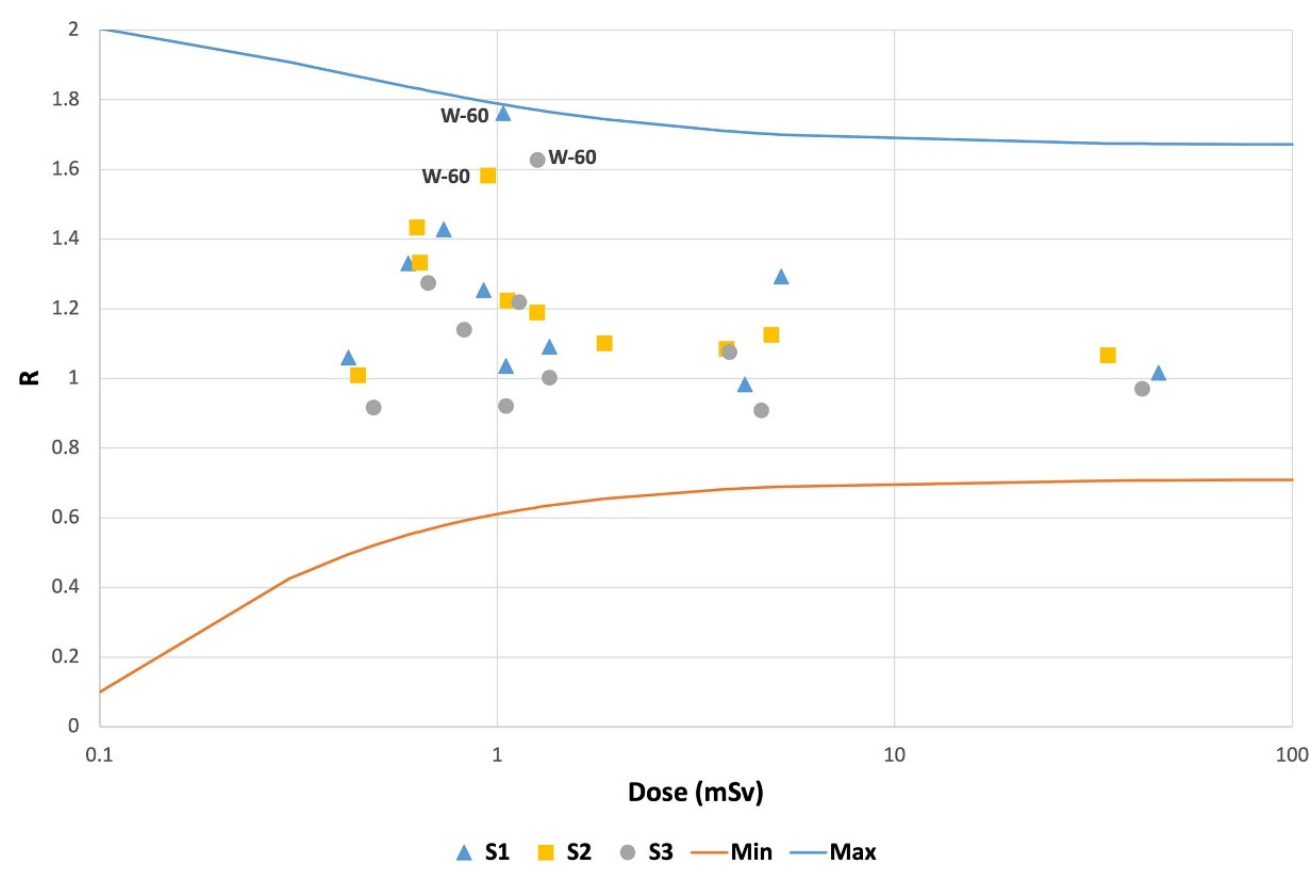

Figure 9. Inter-comparison results in terms of $H_{\mathrm{p}}$ of the two selected IMSs, after the modification of the dosimetric algorithm.

\section{Discussion and conclusion}

The analysis of the whole data set and, in particular, the comparison of figures 3 and 4, shows that the impact of the new dosimetric quantities is mostly on the beam qualities W-60 and W-80, with a mean energy about $45 \mathrm{keV}$ and $54 \mathrm{keV}$, respectively. It is assumed that the effect will be more significant at lower energies. This is an expected result considering the difference in the conversion coefficients for $H_{\mathrm{p}}(10)$ and $H_{\mathrm{p}}$, as reported in table 2, and it agrees with [11], where a similar overestimation at low energies is reported for whole-body dosemeters based on BeOSL technology. The effect of the normalisation coefficient $C_{n}$ has a positive impact, although the response values $R$ are, on average, much larger than unity, indicating an overestimation of the dose assessed by the dosemeter. No particular difference between TLD and film-badge-based dosemeters is noted. Strictly speaking, the results of the hypothetical proficiency test were positive, although the rather large number of points close to the upper limit makes this approach questionable. This means that it is difficult to think of scenario (i) as a reliable option. Scenarios (ii) and (iii) have been checked by asking two IMSs to recalculate their response function. Unfortunately, this second approach does not lead to significant improvement. Comparing figures 8 and 9 it is evident that the tendency towards values $R>1$ is still dominant. Thus, for the two specific dosimetry systems analysed in this work neither scenario (ii) nor scenario (iii) seem to be a valid option. Probably, there is room for finer tuning of the algorithms, but it seems unlikely to obtain a performance in $H_{\mathrm{p}}$ comparable to that in $H_{\mathrm{p}}(10)$, without physical modification of the badge. This means that scenario (iv) cannot be excluded a priori. As mentioned above, the authors are aware that this analysis is limited and does not represent a type test. However, scenario (iv) may have a financial impact on IMSs and, consequently, on the end users. 
If the new operational quantities are accepted by the scientific community, their introduction into national legislation will take 15-20 years. Over this long period, there is time for the scientific community to react, adapting progressively to the new framework. For instance, primary and secondary standard calibration laboratories could provide irradiation certificates reporting both existing and new operational quantities. This can be done without significant additional cost as the irradiation setup is the same. In this way, IMSs can characterise their system in both existing and new quantities. Proficiency test providers can consider exercises similar to the one presented in this work, involving a wider number of dosimetry systems and a wider number of radiation qualities. Such an approach would allow IMSs to gain confidence in the new operational quantities and determine to what extent existing dosemeter badges can be used as 'they are' without reducing the quality of dosimetric results. Alternatively, IMSs can identify the best way to modify the different methods or badge designs to optimise the response to the new operational quantities.

\section{Acknowledgment}

The authors thank Dr Phil Gilvin for his valuable advice on the manuscript.

\section{ORCID iDs}

M Caresana (D) https://orcid.org/0000-0002-6860-7859

R Behrens (D) https://orcid.org/0000-0002-4905-7791

T Otto (D) https://orcid.org/0000-0002-6182-8659

\section{References}

[1] International Commission on Radiation Units and Measurements (ICRU) 2020 Operational Quantities for External Radiation Exposure ICRU Report 95 J. ICRU 20 (Thousand Oaks, CA: Sage Publishing)

[2] Otto T, Hertel N E, Bartlett D T, Behrens R, Bordy J M, Dietze G, Endo A, Gualdrini G and Pelliccioni M 2017 Radiat. Prot. Dosim. 180 10-16

[3] Endo A 2016 Ann. ICRP 45 178-87

[4] ISO G 2019 ISO 4037 radiological protection-x and gamma reference radiation for calibrating dosemeters and doserate meters and for determining their response as a function of photon energy

[5] ISO G 2012 Reference radiation fields for radiation protection definitions and fundamental concepts

[6] Otto T 2019 Response of photon dosimeters and survey instruments to new operational quantities proposed by ICRU RC26 J. Instrument. 1401010

[7] Eakins J and Tanner R 2019 J. Radiol. Prot. 39399

[8] Behrens R and Otto T 2020 J. Radiol. Prot. accepted (available at: http://iopscience.iop.org/article/ 10.1088/1361-6498/abc860)

[9] Online (available at:https://eurados.sckcen.be/) (Accessed 4 November 2020)

[10] 2000 ISO 14146 radiation protection-criteria and performance limits for the periodic evaluation of processors of personal dosemeters for $\mathrm{x}$ and gamma radiation

[11] Hoedlmoser H, Bandalo V and Figel M 2020 Radiat. Meas. 139106482 